\title{
Inhibition of Cyclic 3'-5'-Guanosine Monophosphate-specific Phosphodiesterase Selectively Vasodilates the Pulmonary Circulation in Chronically Hypoxic Rats
}

\author{
Alan H. Cohen, ${ }^{\star}$ Kimberly Hanson, ${ }^{\ddagger}$ Ken Morris, ${ }^{\star}$ Brian Fouty, ${ }^{\star}$ Ivan F. McMurtry, ${ }^{\star}$ William Clarke, ${ }^{\ddagger}$ and David M. Rodman* \\ *Cardiovascular Pulmonary Research Laboratory, University of Colorado Health Sciences Center, Denver, Colorado 80262; \\ and $¥$ University of Washington/Children’s Hospital, Departments of Anesthesia and Pediatrics, Seattle, Washington 98105
}

\begin{abstract}
While it is known that nitric oxide (NO) is an important modulator of tone in the hypertensive pulmonary circulation, the roles of cyclic $3^{\prime}-5^{\prime}$-guanosine monophosphate (cGMP) and cGMP-phosphodiesterase (PDE) are uncertain. We found that isolated lung perfusate levels of cGMP were over ninefold elevated in hypertensive vs. normotensive control rats. 98-100\% of lung cGMP hydrolytic activity was cGMP-specific PDE5, with no significant decrease in PDE activity in hypertensive lungs, suggesting that the elevation in cGMP was due to accelerated production rather than reduced degradation. In pulmonary hypertensive rat lungs, in vitro, cGMP-PDE inhibition by E4021[1-\{6-chloro4-(3,4-methylbenzyl) amino-quinazolin-2-yl\}piperdine-4-carboxylate], increased perfusate cGMP threefold, reduced hypoxic vasoconstriction by $58 \pm 2 \%$, and reduced baseline pulmonary artery pressure by $37 \pm 5 \%$. In conscious, pulmonary hypertensive rats, intravenous administration of E4021 reduced hypoxic vasoconstriction by $68 \pm 8 \%$, pulmonary artery pressure by $12.6 \pm 3.7 \%$ and total pulmonary resistance by $13.1 \pm 6.4 \%$, with no significant effect on cardiac output, systemic pressure, and resistance. Comparison of E4021 to inhaled nitric oxide demonstrated that cGMPPDE inhibition was as selective and as effective as inhaled NO. (J. Clin. Invest. 1996. 97:172-179.) Key words: nitric oxide - Zaprinast e endothelial-derived relaxing factor • cyclic GMP • E4021
\end{abstract}

\section{Introduction}

Pulmonary hypertension encompasses a variety of primary and secondary conditions affecting both children and adults. Common to all forms of the disease is increased vascular tone due to the combined effects of vasoconstriction and vascular wall remodeling. Despite intensive investigation, no generally effective therapy for pulmonary hypertension has been devel-

Portions of this work have appeared in abstract form (1995. Am. J. Respir. Crit. Care Med. 151:626a [Abstr.])

Address correspondence to David M. Rodman, Cardiovascular Pulmonary Research Laboratory, Box B-133, University of Colorado Health Sciences Center, 4200 East Ninth Avenue, Denver, CO 80262. Phone: 303-270-4473; FAX: 303-270-4871; E-mail: rodman_d@ defiance.uchsc.edu

Received for publication 14 June 1995 and accepted in revised form 27 September 1995.

J. Clin. Invest.

(C) The American Society for Clinical Investigation, Inc.

0021-9738/96/01/0172/08 \$2.00

Volume 97, Number 1, January 1996, 172-179 oped. Attempts to develop pulmonary vasodilators have generally been frustrated by a lack of specificity to the pulmonary vasculature. Recently, nitric oxide (NO) has been tested as a selective pulmonary vasodilator in both humans and animals (1-3). Inhaled NO produces significant reductions in pulmonary vascular resistance and pressure, with no concomitant systemic effects (4). The pulmonary selectivity of inhaled NO is conferred by the ability of circulating red blood cell hemoglobin to bind and scavenge NO before it can access the systemic vascular bed. While this therapy holds promise for neonates with rapidly reversible pulmonary hypertension $(5,6)$, its applicability to adults with established pulmonary hypertension is less certain, due to the difficulty and potential toxicity of administering continuous, ambulatory inhaled NO for extended periods of time. Relatively selective pulmonary vasodilation can also be seen during direct intrapulmonary or central venous infusion of a variety of agents with short plasma halflife, including prostacyclin $\left(\mathrm{PGI}_{2}\right)$, L-arginine (in the neonate), and acetylcholine (7-9). While continuous central venous infusion of $\mathrm{PGI}_{2}$ is being applied to therapy of primary pulmonary hypertension, systemic vasodilation continues to limit the efficacy of this approach.

Results from our laboratory and others (10-12) suggest that during the development of pulmonary hypertension, the pulmonary circulation continues to produce NO. Though controversial, most reports in animal models support the hypothesis that NO production may be increased in the hypertensive pulmonary circulation, possibly due to increased nitric oxide synthase (NOS) $)^{1}$ expression (13). A report in newborn lambs has suggested that hypertension-induced NO production can be further enhanced by administration of L-arginine, suggesting that L-arginine may be a selective pulmonary vasodilator in this setting (14). However, two studies in human adults with either systemic sclerosis or primary pulmonary hypertension found no salutary effect of L-arginine infusion on pulmonary pressures but systemic vasodilation in the latter group $(15,16)$.

NO- and atrial natriuretic peptide-mediated pulmonary vasodilation is felt to be mediated by stimulation of smooth muscle cell guanylate cyclase, which catalyzes the cyclization of guanosine triphosphate (GTP) to cyclic 3'-5'-guanosine monophosphate (cGMP). Steady state cellular concentrations of cGMP are established by the balance of production by guanylate cyclase and degradation by phosphodiesterases (PDEs). There are at least seven isoforms of mammalian PDE with varying specificity for hydrolysis of cyclic nucleotides. The cGMP-specific isoform of the PDEs (PDE5) appears to play a significant role in modulating vascular smooth muscle tone (17-19). Recent data suggest that the richest relative source of

1. Abbreviations used in this paper: HPV, hypoxic vasoconstriction; NOS, nitric oxide synthase; PDE, phosphodiesterase; Ppa, pulmonary artery pressure; TPR, total pulmonary resistance. 
PDE5 is the lung and pulmonary vasculature (20), suggesting this enzyme as a potential pharmacological target for pulmonary vasodilation. This concept is supported by reports that Zaprinast, a cGMP-specific PDE inhibitor, can vasodilate the neonatal pulmonary circulation $(8,21)$.

Based on this evidence, in the present study we tested the hypothesis that cGMP-specific PDE regulates pulmonary cGMP content and vascular tone in the chronically hypertensive pulmonary circulation. Specifically we tested in rats the effect of chronic hypoxic pulmonary hypertension on lung cGMP release and cGMP-PDE activity. We also tested the effect of PDE5 inhibition on pulmonary vascular tone and hypoxic pulmonary vasoconstriction in lungs from chronically hypoxic rats both in vitro and in vivo. To determine the degree of pulmonary specificity, we measured pulmonary and systemic hemodynamics in vivo in chronically hypoxic rats during PDE5 inhibition, comparing these results to rats treated with clinically relevant doses of either inhaled NO or calcium channel blocker.

\section{Methods}

Animals. Male adult Sprague-Dawley rats (250-450 g) were used in all experiments. "Low altitude" rats (pulmonary normotensive controls) were kept at Denver's altitude of 5,280 ft. High altitude rats (chronically hypoxic pulmonary hypertensive) were exposed to a simulated altitude of $17,000 \mathrm{ft}$ in a hypobaric chamber which was flushed continuously with room air to prevent accumulation of $\mathrm{CO}_{2}, \mathrm{NH}_{3}$, and water vapor. The high altitude rats were exposed to 12-h light/ dark cycle and allowed free access to standard rat food and water in hypobaric hypoxia for $24 \mathrm{~h} / \mathrm{d}$ for 3-5 wk before experiments were performed. In vivo hemodynamic measurements were made within $6 \mathrm{~h}$ of removal from hypobaric hypoxia.

Isolated perfused rat lungs. As previously described (10), lungs were isolated from low and high altitude rats after intraperitoneal administration of $30 \mathrm{mg}$ pentobarbital and intracardiac injection of 100 IU heparin. Isolated lungs were ventilated with a humid mixture of $21 \% \mathrm{O}_{2}+5 \% \mathrm{CO}_{2}+74 \% \mathrm{~N}_{2}$ at a rate of 60 breaths/min and at an inspiratory pressure of $9 \mathrm{~cm} \mathrm{H}_{2} \mathrm{O}$ and an end expiratory pressure of 2.5 $\mathrm{cm} \mathrm{H}_{2} \mathrm{O}$ using a rodent ventilator (Harvard Apparatus Inc., South Natick, MA). The heart-lung preparations were perfused through a main pulmonary artery cannula by a peristaltic pump at a constant flow of $0.04 \mathrm{ml} / \mathrm{g}$ body wt per min. The perfusate was a physiological salt solution (PSS [Earle's Balanced Salts without phenol red and sodium bicarbonate] Sigma Chemical Co., St. Louis, MO) composed of (in g/liter): $0.265 \mathrm{CaCl}_{2} \cdot 2 \mathrm{H}_{2} \mathrm{O}, 0.09767 \mathrm{MgSO}_{4}, 0.4 \mathrm{KCl}, 6.8 \mathrm{NaCl}$, $0.122 \mathrm{NaH}_{2} \mathrm{PO}_{4}$, and $1.0 \mathrm{D}$-glucose. Ficoll (4 g/100 ml, type 70, Sigma Chemical Co.) was included as a colloid, sodium meclofenamate (3.1 $\mu \mathrm{M})$ to inhibit cyclooxygenase (Warner-Lambert Pharmaceutical Co., Morris Plains, $\mathrm{NJ}$ ), and $\mathrm{NaHCO}_{3}(1.6 \mathrm{~g} /$ liter) to adjust $\mathrm{pH}$ to the physiologic range. After the lungs were flushed free of blood, with 20 $\mathrm{ml}$ of PSS, they were perfused with a recirculated vol of $30 \mathrm{ml}$. Effluent perfusate drained from a left ventricular cannula into the perfusate reservoir. Lung and perfusate temperatures were maintained at $38^{\circ} \mathrm{C}$, and perfusate $\mathrm{pH}$ was kept between 7.35 and 7.45. Mean perfusion pressure was measured continuously with a transducer and pen recorder, and the perfused lungs were equilibrated for $20 \mathrm{~min}$ before vascular responses were measured. The various drugs used in the specific protocols described below were added to the perfusate reservoir to achieve calculated circulating concentrations.

Measurement of perfusate cGMP. As previously described (10), five normotensive and five pulmonary hypertensive rat lungs were prepared and perfused with PSS perfusate using the isolated-perfused model. Four, 500- $\mu \mathrm{l}$ samples of perfusate were sequentially collected and immediately added to $30-\mu l$ of isobutyl methyl xanthin $\left(5 \times 10^{-4} \mathrm{M}\right.$, final concentration) before being quick frozen in liquid nitrogen. After 20 min of equilibration and normoxic ventilation, the first baseline sample was collected. The second sample was drawn after three (10min) hypoxic challenges $\left(0 \% \mathrm{O}_{2}, 5 \% \mathrm{CO}_{2}, \mathrm{~N}_{2}\right.$ balanced). The third and fourth samples were then collected at $30 \mathrm{~min}$ intervals, before completion of each experiment. The frozen PSS-perfusates were then assayed using a Biomedical Technologies Inc. (Stoughton, MA) cyclic GMP RIA system. It uses a preconjugated double antibody separation system in an acetate buffer. Duplicate samples were performed on all samples, including the standard curve, and all values are calculated and reported as picomoles per milliliter of cGMP.

Measurement of PDE lung activity. Lung samples were removed and placed in homogenate buffer (40 mM Tris-HCI, $\mathrm{pH} 7.5,15 \mathrm{mM}$ benzamidine, $15 \mathrm{mM}$ 2-mercaptoethanol, $1 \mu \mathrm{g} / \mathrm{ml}$ pepstatin A, $1 \mu \mathrm{g} /$ $\mathrm{ml}$ leupeptin, $5 \mathrm{mM}$ EDTA, $1 \mathrm{mM}$ PMSF, and $25 \mu \mathrm{g} / \mathrm{ml}$ antipain) at a ratio of 1:4. Samples underwent homogenization at $4^{\circ} \mathrm{C}$. The samples were centrifuged at $1,000 \mathrm{~g}$ for $10 \mathrm{~min}$. The supernatant was removed and centrifuged at 100,000 $\mathrm{g}$ for $1 \mathrm{~h}$. The supernatant was removed, pellets were Dounce homogenized, and all samples were placed in $20 \%$ glycerol and stored at $-70^{\circ} \mathrm{C}$. A modification of the method of Beavo et al. (22) was used for calmodulin-independent assays of PDE activity. The buffer contained $40 \mathrm{mM}$ Mops, $\mathrm{pH}$ 7.5, $0.8 \mathrm{mM}$ EGTA, $15 \mathrm{mM}$ MgAOc, $0.2 \mathrm{mg} / \mathrm{ml}$ BSA, $1 \mu \mathrm{M}$ cold cGMP, $10^{5} \mathrm{cpm}$ hot cGMP, and $4 \mathrm{uM}$ Zaprinast or no inhibitor added. The reaction was initiated by adding the extract (final reaction vol of $250 \mu \mathrm{l}$ ). The samples were incubated at $30^{\circ} \mathrm{C}$ for $10 \mathrm{~min}$. The reaction was stopped by boiling the sample for $1 \mathrm{~min}$. The reaction was cooled and $10 \mu \mathrm{l}$ of 2.5 $\mathrm{mg} / \mathrm{ml}$ of Crotalus atrox snake venom (Sigma Chemical Co.) was added and incubated for $5 \mathrm{~min}$ at $30^{\circ} \mathrm{C}$. The reaction was diluted with $250 \mu \mathrm{l}$ of low salt buffer ( $20 \mathrm{mM}$ Tris- $\mathrm{HCl}, \mathrm{pH} 7.5,0.1 \%$ Tween 20 ) and applied to A-25 ion exchange resin column. The columns were washed four times with low salt buffer and the column volume was collected in scintillation vials. The vials were filled with $5 \mathrm{ml}$ of scintillation cocktail and counted for $1 \mathrm{~min}$. The proteins were determined by the method of Bradford (23).

Conscious catheterized rats. As previously described (10), rats were anesthetized with intramuscular Ketamine $(100 \mathrm{mg} / \mathrm{kg}$; Fort Dodge Laboratories, Fort Dodge, IA) and Rompun (15 mg/kg; Miles, Shawnee, KS) for placement of catheters in the pulmonary and right carotid arteries, as well as the right jugular vein. The intravascular location of the catheter tips were determined by the blood pressure tracings, and the catheters were secured, filled with heparinized saline containing $1 \mathrm{mg} / \mathrm{ml}$ chloramphenicol, sealed, and tunneled subdermally to the back of the neck where they were exteriorized and enclosed in a small plastic container. The incisions were closed, and the rats were allowed to recover for $24 \mathrm{~h}$ in room air at low altitude. After recovery, the conscious rat was placed in a ventilated clear plastic chamber for measurement of pulmonary and systemic blood pressures and cardiac output. Pulmonary and systemic arterial pressures were measured with transducers (Statham Instruments, Hato Rey, PR) and mean pressures were calculated by computer. Cardiac output was measured by standard dye-dilution technique. As expected, mean pulmonary arterial pressure (Ppa) in the hypoxic group $(n=$ 10) measured after $3-4 \mathrm{wk}$ of hypobaric hypoxia was $41.1 \pm 2.3$ $\mathrm{mmHg}$, and $19.7 \pm 0.6 \mathrm{mmHg}$ in the normoxic rats $(n=9)$.

Experimental protocols. In vitro experiments examined the effects of the PDE5 inhibitor E4021 (Eisai Co., Ibaraki, Japan), on baseline perfusion pressure and acute hypoxic vasoconstriction in PSS-perfused high altitude lungs. In vivo, the $\mathrm{IC}_{50}$ of E4021 for PDE5 is $3.5 \mathrm{nM}$, whereas the $\mathrm{IC}_{50}$ for PDEs $1-4$ is $\geq 23 \mu \mathrm{M}$ (24). Therefore, for all experiments the maximum concentration of E4021 used (10 $\mu \mathrm{M})$ predominantly inhibited PDE5. After 20 min of equilibration, the isolated lungs were challenged three times with airway hypoxia at 5-min intervals. $5 \mathrm{~min}$ after the third hypoxic pressor response, logarithmically increasing doses of either E4021 or vehicle were added to the perfusate $\left(10^{-11}-10^{-5} \mathrm{M}\right)$, and after $5 \mathrm{~min}, 10$-min hypoxic challenges were performed.

After these in vitro preliminary studies, we compared the pulmonary and systemic hemodynamic effects of E4021 to infused Zaprinast, diltiazem (Sigma Chemical Co.) or inhaled nitric oxide (Airco, 
Murray Hill, NJ) in conscious catheterized rats. After measuring resting (normoxic, fraction of inspired oxygen $=0.21$ ) pulmonary and systemic arterial pressures and cardiac output, normotensive and hypertensive rats were exposed to two, 5-6 min of hypoxia (fraction of inspired oxygen $=0.10$ ), and hemodynamic measurements were repeated. Blood $\mathrm{pH}$ measurements were taken before and during administration of the E4021 and during vehicle studies with no change in $\mathrm{pH}$ noted (Radiometer ABL2; Acid Base Laboratory, Copenhagen, Denmark).

Drugs and chemicals. For in vitro studies E4021 was dissolved in DMSO (Sigma Chemical Co.). For in vivo studies E4021 was prepared as a $10^{-3} \mathrm{M}$ solution in $0.01 \mathrm{~N} \mathrm{NaOH}$ and gently heated. Diltiazem was readily soluble in normal saline and Zaprinast was mixed in $0.05 \mathrm{~N} \mathrm{NaOH}$. E4021 as well as Zaprinast and diltiazem were injected intravenously into an internal jugular vein catheter, as a bolus, followed by continuous infusion using an infusion pump (Harvard Apparatus, Inc.) (doses detailed in the legend to Table I). Hemodynamic measures were made, both during normoxia and hypoxic challenges and after drug infusion. Vehicle controls were performed for 0.05 and $0.01 \mathrm{~N} \mathrm{NaOH}$ following the same protocol. A similar protocol was performed using inhaled NO. For experiments requiring inhaled $\mathrm{NO}$, the appropriate $\mathrm{NO} / \mathrm{N}_{2} / \mathrm{O}_{2}$ mixture was introduced to the chamber and $\mathrm{NO}$ and $\mathrm{O}_{2}$ levels were monitored with chemiluminescence (270B; Sievers, Boulder, $\mathrm{CO}$ ) and an $\mathrm{O}_{2}$ fuel cell (Applied Technical Products, Denver, CO), respectively.

Data analysis. Total pulmonary resistance (TPR) was calculated by dividing mean Ppa by cardiac output. Total systemic resistance was calculated by dividing mean systemic pressure by cardiac output. Statistical analysis was done by Student's $t$ test or ANOVA with Fisher post hoc test. Differences were considered significant at $P<$ 0.05 . Data are presented as mean \pm SEM.

\section{Results}

Fig. 1 shows the results of perfusate cGMP measurements in normotensive $(n=5)$ and pulmonary hypertensive $(n=5)$ isolated perfused rat lungs. There was a time-dependent accumulation of cGMP in the perfusate which was significantly greater in the hypertensive group. At the time of study termination,

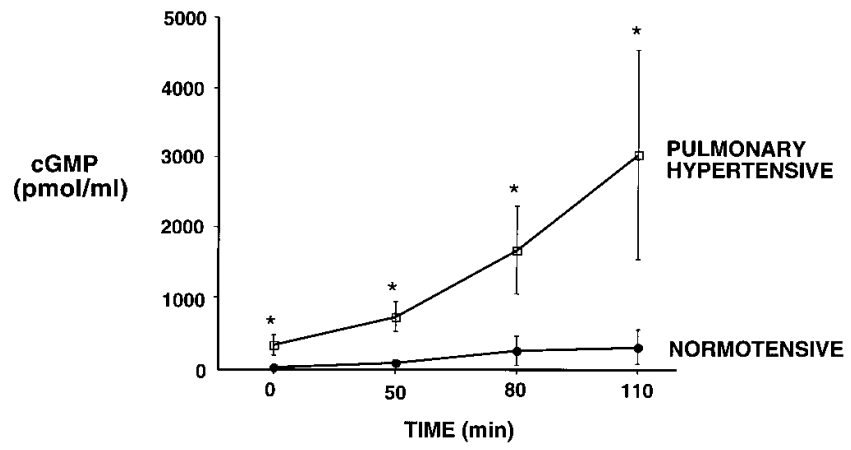

Figure 1. Perfusate cGMP measurements in normotensive $(n=5)$ and pulmonary hypertensive $(n=5)$ isolated perfused rat lungs.

There were significantly greater levels of cGMP measured at all time points in the pulmonary hypertensive group, relative to normotensive control lungs $* P<0.05$ from control.

the mean perfusate cGMP level in the hypertensive group was over ninefold greater than that in the control group.

Studies measuring PDE activity from lung tissue of normotensive $(n=5)$ and hypertensive $(n=5)$ rats showed that total cGMP PDE hydrolytic activity tended to be slightly, though not significantly, lower in pulmonary hypertensive rat lungs (Fig. 2). Zaprinast-inhibitable PDE activity was the predominate isotype in both groups, with percent inhibition of PDE activity by Zaprinast of 98 and $100 \%$, in normotensive and hypertensive lungs, respectively.

Based on these findings, we next tested the hypothesis that inhibition of cGMP-specific PDE5 would potentiate endogenous cGMP-mediated dilation of the pulmonary vascular bed. To confirm the activity of the novel PDE5 inhibitor E4021 in rat lung, either $100 \mathrm{nM}$ E4021 $(n=4)$ or vehicle $(n=6)$ was added to the perfusate reservoir of hypertensive rat lungs, and perfusate cGMP measured 40 min later compared to baseline.

Table I. Measurements of Ppa, Carotid/Systemic Artery Pressure, and Cardiac Output Are Reported in Catheterized Chronically Pulmonary Hypertensive Rats

\begin{tabular}{|c|c|c|c|c|c|c|}
\hline & HPV & Ppa & TPR & Pca & TSR & $\mathrm{CO}$ \\
\hline \multicolumn{7}{|l|}{ E4021 } \\
\hline Vehicle control $(n=5)$ & $-0.3 \pm 18.3$ & $9.8 \pm 3.8$ & $-2.9 \pm 6.2$ & $0.9 \pm 6.9$ & $0.5 \pm 6.8$ & $16.0 \pm 8.7$ \\
\hline Low dose $(n=10)$ & $-68.5 \pm 8^{*}$ & $-12.6 \pm 3.7^{*}$ & $13.1 \pm 6.4^{*}$ & $2.3 \pm 3.0$ & $0.3 \pm 5.9$ & $5.6 \pm 7.4$ \\
\hline High dose $(n=4)$ & $-78.2 \pm 5.3^{*}$ & $-8.2 \pm 6.0$ & $-14.4 \pm 8.0$ & $-15.1 \pm 2.8$ & $-19.6 \pm 9.8$ & $11.8 \pm 17.7$ \\
\hline \multicolumn{7}{|l|}{ Zaprinast } \\
\hline Low dose $(n=4)$ & $-102.3 \pm 14^{*}$ & $-13.2 \pm 3.6^{*}$ & $-7.3 \pm 7.9$ & $-8.4 \pm 3.6$ & $-2.2 \pm 8.7$ & $-4.7 \pm 7.3$ \\
\hline High dose $(n=4)$ & $-93.6 \pm 5.9 *$ & $-13.0 \pm 8.1$ & $8.5 \pm 6.5$ & $-27.1 \pm 4.9^{*}$ & $-7.1 \pm 8.1$ & $-18.7 \pm 9.5$ \\
\hline \multicolumn{7}{|l|}{ Diltiazem } \\
\hline Low dose $(n=5)$ & $-78.0 \pm 6.8^{*}$ & $-4.2 \pm 5.5$ & $-8.2 \pm 6.2$ & $-11.3 \pm 3.3 *$ & $-15.6 \pm 4.7^{*}$ & $6.7 \pm 7.6$ \\
\hline High dose $(n=6)$ & $-82.4 \pm 12.3$ & $-13.0 \pm 5.9$ & $23.1 \pm 13.1$ & $-32.7 \pm 4.0$ & $-4.4 \pm 11.7$ & $-23.2 \pm 11.4$ \\
\hline \multicolumn{7}{|l|}{ Nitric oxide } \\
\hline $10 \operatorname{ppm}(n=3)$ & $-32.2 \pm 11.8^{*}$ & $-4.1 \pm 7.4$ & $6.5 \pm 4.9$ & $5.4 \pm 7.1$ & $17.4 \pm 6.3$ & $10.2 \pm 3.7$ \\
\hline $40 \operatorname{ppm}(n=3)$ & $-46.3 \pm 13.7 *$ & $-9.2 \pm 9.9$ & $-3.4 \pm 9.4$ & $2.8 \pm 3.1$ & $10.1 \pm 5.7$ & $-6.3 \pm 3.9$ \\
\hline $80 \mathrm{ppm}(n=5)$ & $-56.1 \pm 15.2 *$ & $-14.6 \pm 3.5^{*}$ & $-1.2 \pm 5.2$ & $-0.7 \pm 2.1$ & $15.4 \pm 7.0$ & $-13.0 \pm 3.7 *$ \\
\hline
\end{tabular}

HPV to $10 \%$ oxygen is measured. TPR and total systemic resistance (TSR) are calculated. Values are mean percent changes \pm SEM. Negative values $(-)$ represent decreases and positive values increases in mean. Drug doses used: E4201, vehicle control $(0.01 \mathrm{~N} \mathrm{NaOH})$, low dose $(300 \mu \mathrm{g} / \mathrm{kg}+0.01$ $\mathrm{mg} / \mathrm{min})$, high dose $(1.2 \mathrm{mg} / \mathrm{kg}+0.04 \mathrm{mg} / \mathrm{min}) ;$ Zaprinast, low dose $(1 \mathrm{mg} / \mathrm{kg})$, high dose $(20 \mathrm{mg} / \mathrm{kg}+0.28 \mathrm{mg} / \mathrm{min}) ;$ diltiazem, low dose $(1 \mathrm{mg} / \mathrm{kg}+$ $0.02 \mathrm{mg} / \mathrm{min})$, high dose $(2 \mathrm{mg} / \mathrm{kg}+0.04 \mathrm{mg} / \mathrm{min})$. *Significant values $(P<0.05)$. Pca, carotid/systemic artery pressure; CO, cardiac output. 


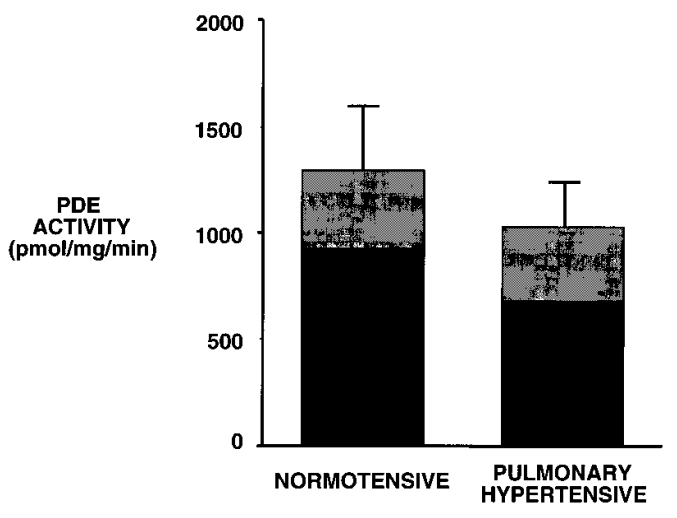

Figure 2. Measurements of cGMP hydrolytic PDE activity from homogenized lung tissue of normotensive $(n=5)$ and pulmonary hypertensive $(n=5)$ rats showed no significant difference between the groups. The cGMP PDE hydrolytic activity of the supernatant is represented in gray, the particulate activity is denoted in black.

The increase in perfusate cGMP was $3,476 \pm 331 \mathrm{pM} / \mathrm{ml}$ in E4021-treated lungs vs. $1,166 \pm 354 \mathrm{pM} / \mathrm{ml}$ in control lungs $(P<$ $0.01)$. The pulmonary effects of cGMP-specific PDE inhibition were then studied by adding E4021 in logarithmically increasing doses into the PSS perfusates of chronically hypoxic, hypertensive rat lungs. Fig. 3 shows that compared to vehicle, E4021 reduced mean Ppa by $33.3 \pm 9.0$ and $37.5 \pm 5.1 \%$ at concentrations of $10^{-6} \mathrm{M}$ and $10^{-5} \mathrm{M}$, respectively $(n=3)$. As illustrated in Fig. 4, E4021 also reduced the mean hypoxic pressor response by $47.2 \pm 2.8$ and $57.9 \pm 2.2 \%$ at these concentrations $(n=3)$. Inhibition $(10.5 \pm 6.6 \%, P<0.05)$ of the hypoxic pressor response was also seen at an E4021 concentration as low as $10^{-9} \mathrm{M}$. Based on these preliminary findings, in vivo studies were then performed.

Initially, in vivo studies were performed in catheterized and conscious normotensive rats. As illustrated in Fig. 5, at a loading dose of $300 \mathrm{mg} / \mathrm{kg}$ and continuous infusion of $0.01 \mathrm{mg} / \mathrm{min}$, E4021 reduced hypoxic pulmonary vasoconstriction (HPV) in normotensive rats $(n=9)$ by $66.1 \pm 8.1 \%$. As expected, this same "low dose" of E4021 had no significant effect on the already low Ppa and did not affect systemic pressure. Subse-

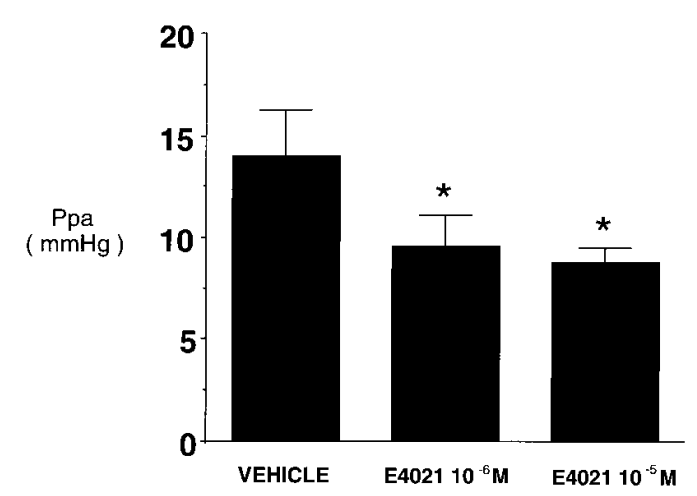

Figure 3. Effect of E4021, a specific and selective PDE5 inhibitor, on baseline pulmonary artery perfusion pressure (Ppa) compared to vehicle control (DMSO) in chronically hypoxic, pulmonary hypertensive isolated rat lungs. E4021 significantly reduced the Ppa at concentrations of $10^{-6} \mathrm{M}$ and $10^{-5} \mathrm{M}(n=3)$. $* P<0.05$ from control.

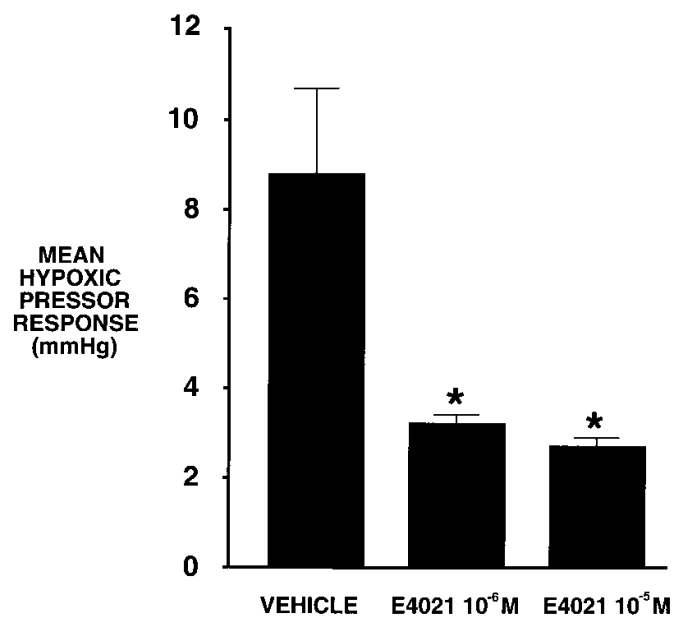

Figure 4. Effect of PDE5 inhibition by E4021, on the mean hypoxic pressor response in chronically hypoxic, pulmonary hypertensive isolated rat lungs. E4021 significantly reduced hypoxic pulmonary vasoconstriction, in response to $0 \%$ oxygen, at concentrations of $10^{-6} \mathrm{M}$ and $10^{-5} \mathrm{M}$, relative to vehicle control. $* P<0.05$ from control.

quent studies, performed in 10 catheterized and conscious pulmonary hypertensive rats (Fig. 6) demonstrated that low dose E4021 (300 $\mu \mathrm{g} / \mathrm{kg}$ load then $0.01 \mathrm{mg} / \mathrm{min}$ infusion) reduced the baseline Ppa by $12.6 \pm 3.7 \%$, relative to a rise of $9.8 \pm 3.8 \%$ in the vehicle group $(n=6)$. As illustrated in Fig. 7, 8 of 10 animals showed a fall in Ppa during E4021 infusion, and a similar reduction of $13.1 \pm 6.4 \%$ was seen in calculated total pulmonary resistance (TPR). In contrast, 8 of 9 animals showed no change in systemic pressure and total systemic resistance, and significant changes in cardiac output were not seen (Table I). In addition to reducing Ppa, E4021 attenuated hypoxic pulmonary vasoconstriction to $10 \%$ oxygen (Fig. 5), by $68.5 \pm 8.0 \%$.

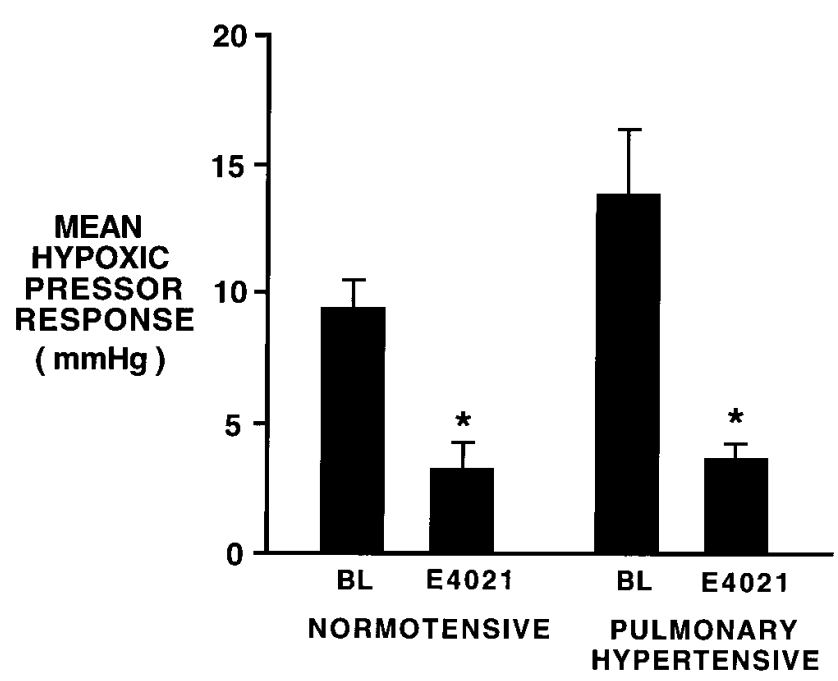

Figure 5. In catheterized and conscious normotensive $(n=9)$ and chronically hypoxic pulmonary hypertensive rats $(n=10)$ E4021 significantly reduced the mean hypoxic pressor response to $10 \%$ oxygen Doses of E4021 were administered as a loading dose of $300 \mu \mathrm{g} / \mathrm{kg}$ and continuous infusion of $0.01 \mathrm{mg} / \mathrm{min}$ (low dose). $* P<0.05$ from control. $B L$, baseline. 


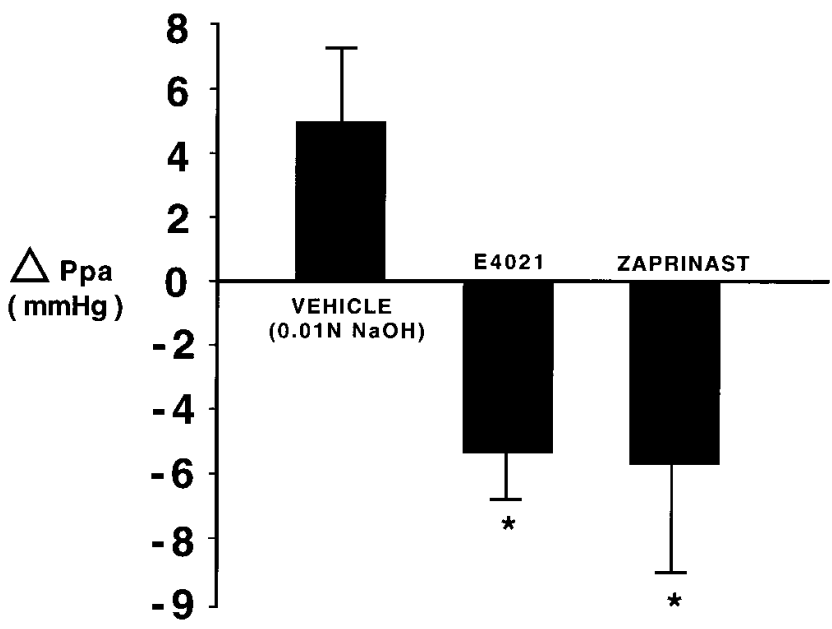

Figure 6. In catheterized and conscious pulmonary hypertensive rats both cGMP-PDE inhibitors, E4021 $(n=10)$ and Zaprinast $(n=4)$, significantly reduced the baseline Ppa relative to vehicle control $(n=$ 6). Low dose E4021 (300 $\mu \mathrm{g} / \mathrm{kg}$ load then $0.01 \mathrm{mg} / \mathrm{min}$ infusion) and Zaprinast (1 mg/kg load) both reduced the Ppa, relative to a rise in the vehicle group. ${ }^{*} P<0.05$ from vehicle control.

We next tested if this was a class effect of cGMP-specific PDE inhibitors. Zaprinast, a chemically distinct but less specific and potent cGMP-PDE inhibitor, at a loading dose of 10 $\mathrm{mg} / \mathrm{kg}$ with an infusion of $0.14 \mathrm{mg} / \mathrm{min}$, produced a reduction in baseline Ppa of $13.2 \pm 3.6 \%(n=4)$. This was comparable to the reduction in Ppa produced by E4021, but at $>30 \times$ the loading dose of E4021 (Fig. 8). Zaprinast, at both doses studied, also effectively blunted HPV in hypertensive rats (Table I) in a manner comparable to that of E4021. A modest fall in systemic pressure was seen at the higher dose of Zaprinast.

Dose responses were then performed in hypertensive rats, to compare diltiazem, a calcium channel blocker, to the pulmonary vasodilatory effects of the PDE inhibitors. At both concentrations studied, diltiazem effectively reduced HPV (Table I), but at the higher dose $(n=6)$, which produced a comparable drop in Ppa to that of E4021 and Zaprinast, there was a significant reduction in systemic pressure (Fig. 8) and cardiac output (Table I).
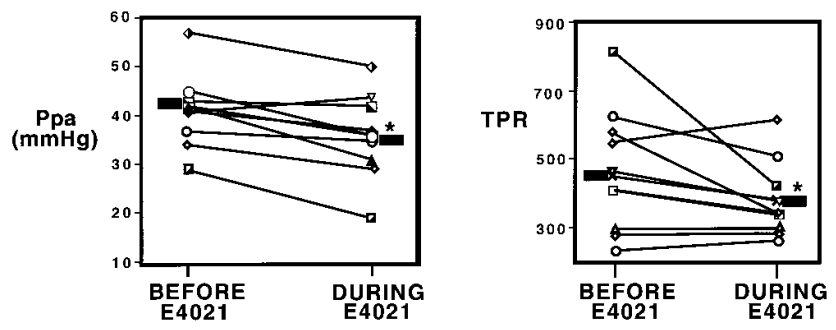

Figure 7. Pulmonary vasodilator response in hypoxic pulmonary hypertensive rats, in vivo, in response to E4021. Symbols represent determinations in 10 individual rats of measured Ppa and calculated TPR either before or during administration of drug. Solid horizontal bars represent group mean values. During E4021 infusion, Ppa fell by $0-35 \%$ (mean $12.6 \pm 3.7 \%$ ) in 8 of 10 rats, and TPR fell by $0-47 \%$ (mean $13.1 \pm 6.4 \%$ ). While there was significant interanimal variability, decreases in group means were significant for both Ppa and TPR $(* P<0.05)$ during E4021 infusion.

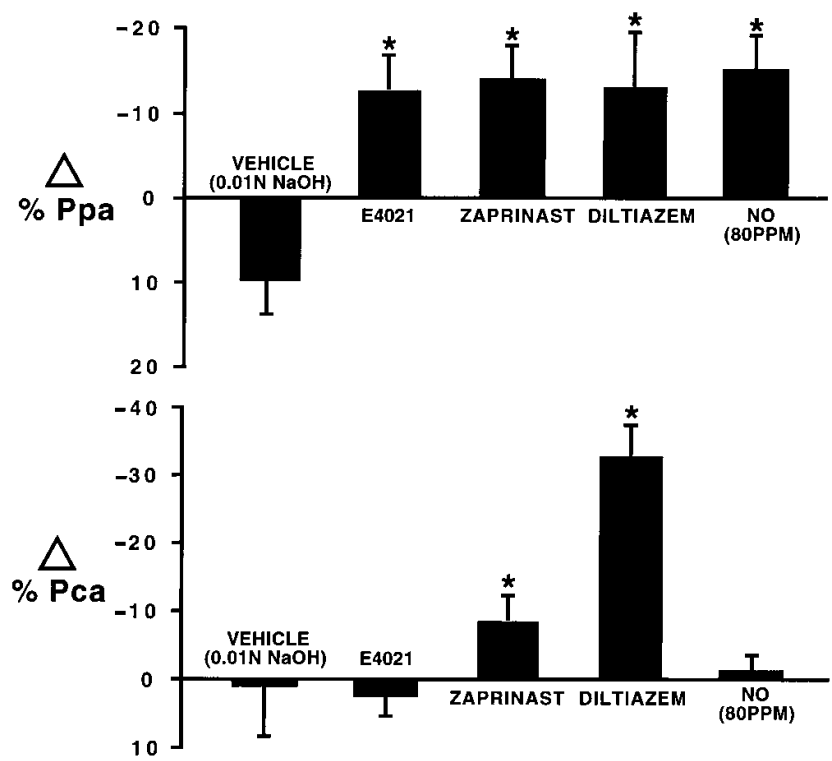

Figure 8 . In conscious, catheterized pulmonary hypertensive rats, only low dose E4021 (300 $\mu \mathrm{g} / \mathrm{kg}$ load plus $0.01 \mathrm{mg} / \mathrm{min}$ infusion) and inhaled nitric oxide $(\mathrm{NO})$, at $80 \mathrm{ppm}$, produced significant $(P<0.05)$ and maximal percent reduction in pulmonary artery pressure $(\Delta \%$ Ppa) with no significant percent change in systemic pressure $(\Delta \%$ Pca). High dose Zaprinast $(n=4)(20 \mathrm{mg} / \mathrm{kg}+0.28 \mathrm{mg} / \mathrm{min}$ infusion $)$ and diltiazem $(n=6) 2 \mathrm{mg} / \mathrm{kg}+0.04 \mathrm{mg} / \mathrm{min}$ also produced maximal, comparative pulmonary artery dilation, but with significant systemic dilation $(\Delta \%$ Pca). $* P<0.05$ from control.

The effects of inhaled NO were then studied in conscious and catheterized hypertensive rats at doses of 10,40 , and 80 ppm. Once again, all three concentrations of inhaled NO effectively blunted HPV, but only the highest dose studied $(n=5)$ comparably dilated the pulmonary vascular bed, by $14.6 \pm 3.5 \%$, to that of the PDE inhibitors (Table I). At $80 \mathrm{ppm}$ there were no significant changes in systemic pressure, although a modest fall in cardiac output was seen.

\section{Discussion}

Our group and others have found that in the normotensive pulmonary circulation of a variety of species, including humans, modulation of pulmonary vasoconstriction is partially dependent upon endogenous NO production $(25,26)$. During the establishment of pulmonary hypertension, NO assumes a central role in modulating the development of increased vascular tone $(10,11)$. Isaacson et al. (12), in hypoxic pulmonary hypertensive rats, measured the NO oxidation product nitrite in lung perfusate as an index of NO production, finding an increase from $0.4 \mathrm{nM}$ in normotensive controls to $24.3 \mathrm{nM}$ in pulmonary hypertensive rats. We found a comparable increase in lung perfusate total nitrate plus nitrite in our hypoxic pulmonary hypertensive rats (unpublished observation). Both our group and Isaacson et al. found that the increased NO was modulating pulmonary vasoconstriction, as inhibition of NOS by L-arginine analogues produced a $20-40 \mathrm{mmHg}$ increase in Ppa. While the mechanisms of NO-mediated pulmonary vasodilation in the pulmonary hypertensive vasculature have not been fully elucidated, it has generally been assumed that stim- 
ulation of vascular smooth muscle cell guanylate cyclase and cGMP production is the principal mechanism of NO's action. In this study we confirmed a likely role for the second messenger cGMP, as we found over a ninefold increase in perfusate cGMP from hypertensive lungs relative to normotensive controls.

While the increase in cGMP was consistent with increased stimulation of guanylate cyclase, a decrease in endogenous cGMP-PDE activity could also contribute to accelerated cGMP accumulation. We therefore measured PDE activity in hypertensive and normotensive rat lungs. We found that lung cGMP PDE hydrolytic activity was largely Zaprinast inhibitable, suggesting that much of this activity was mediated by cGMP-specific PDE5 (20). While there was a trend towards reduced total cGMP PDE activity in hypertensive rat lungs, the magnitude was small and unlikely to account for the substantial elevation in perfusate cGMP. Thus, since significant decreases in cGMP degradation were not seen, it appears that the principal mechanism of increased cGMP in the hypertensive rat lung is increased cGMP production.

The distribution of PDEs appears to be tissue specific. Cyclic GMP-specific PDEs are concentrated in the retina, platelets, and lung (20). Several studies in the isolated lung circulation have confirmed a role for PDE5 in modulating pulmonary artery smooth muscle cell tone during acute vasoconstriction $(8,27)$. In contrast, in the systemic circulation, it appears that cGMP-PDE may be less important than cAMP-PDEs, particularly milrinone-inhibitable PDE3 $(18,28)$. In addition, while amrinone and milrinone can dilate the constricted pulmonary bed, in vitro, these agents lacks pulmonary selectivity (29). Given the apparent up-regulation in pulmonary hypertension of lung cGMP production, the preservation of cGMP-specific phosphodiesterase activity in hypertensive lung, and the tissue specificity of PDEs, we hypothesized that inhibition of cGMPPDE would result in relatively specific vasodilation of the hypertensive pulmonary circulation.

Previous in vivo studies of cGMP-PDEs have used either Zaprinast or dipyridamole. While these two agents are cGMPPDE inhibitors, they lack sufficient potency and specificity to be ideal pharmacological tools. Zaprinast in micromolar concentrations inhibits PDE1, PDE2, and PDE4 at $\mathrm{IC}_{50} \mathrm{~s}$ which are only one to two orders of magnitude greater than the $\mathrm{IC}_{50}$ for PDE5 (24). Thus at pharmacological doses, Zaprinast can inhibit cAMP breakdown. In addition to PDE5 inhibition, dipyridamole is an adenosine reuptake inhibitor. As adenosine may directly and indirectly stimulate cyclic nucleotide production, this property of dipyridamole may complicate interpretation of its effects (21). We therefore used a newly described PDE5 inhibitor, E4021, which has an $\mathrm{IC}_{50}$ for PDE5 which is $3.5 \mathrm{nM}$ (vs. $450 \mathrm{nM}$ for Zaprinast) and has no detectable inhibition of PDE1, PDE3, and PDE4 (24). While E4021 does have some PDE2 inhibitory activity, the $\mathrm{IC}_{50}$ for PDE2 is four orders of magnitude greater than for PDE5.

In isolated perfused lungs from chronically hypoxic rats, E4021 caused dose-dependent inhibition of hypoxic vasoconstriction. This effect is consistent with prior reports that acute HPV is modulated by and may induce NO production in the rat lung (24). In concert with the blunting of HPV, E4021 reduced perfusion pressure by $33 \%$. As this experiment was performed at constant flow, the reduction in perfusion pressure was indicative of reduced pulmonary vascular resistance during cGMP-PDE inhibition. These results are consistent with those previously reported by Clarke et al. (29) in rabbit lungs, and Rosenkrantz et al. (30) and Mlczoch et al. (31) in pigs and dogs, respectively. In these prior studies the less specific PDE5 inhibitor dipyridamole inhibited U46619 and hypoxic vasoconstriction in lungs from normotensive animals.

To determine whether cGMP-PDE inhibition would vasodilate the pulmonary circulation in vivo, several studies were undertaken in conscious, catheterized rats. In normotensive control rats E4021 infusion caused a dose-dependent inhibition of hypoxic vasoconstriction, suggesting enhancement of endogenous pulmonary NO/cGMP-mediated modulation of acute pulmonary vasoconstriction. No effect on basal pulmonary vascular tone was seen. This was anticipated, as the normal rat circulation is already maintained at low tone through NO-independent mechanisms (24). However, in a report in normal pigs, which, like humans, have a higher degree of active resting pulmonary tone, E4021 reduced mean pulmonary artery pressure by $21 \%$, confirming its pulmonary vasodilatory capacity (32).

In pulmonary hypertensive animals, E4021 caused comparable attenuation of HPV to that seen in normotensive controls, and in addition a dose-dependent decrease in both pulmonary artery pressure and total pulmonary resistance. Maximum reduction in pulmonary artery pressure of $13 \%$ was seen at the lowest dose of E4021 which was tested. This degree of maximum pulmonary vasodilation is comparable to that which we have previously seen in this model (33). While no effects on systemic pressure, total systemic resistance, or cardiac output were seen at this dose, after a fourfold increase in dose, modest decreases in systemic pressure and resistance were seen. The failure of higher dose E4021 to further dilate the pulmonary circulation was likely due to having achieved maximal pulmonary vasodilation at the lower dose. While the mechanism of systemic vasodilation at the higher dose is uncertain, it may be due to inhibition at the higher dose of PDE2. Alternatively, it is possible that more complete inhibition of PDE5 in the systemic circulation was achieved at the higher dose of E4021.

While we are aware of no prior studies of PDE5 inhibition in established pulmonary hypertension, two prior studies tested the effect of dipyridamole on pulmonary hemodynamics in adult animals. Rosenkrantz et al. found that dipyridamole inhibited acute hypoxic vasoconstriction in pigs (30). While this observation was attributed to antiplatelet effects, Mlczoch et al. made a similar observation in platelet-depleted dogs (28). In that study the confounding effects on adenosine reuptake were presumed to be responsible for attenuation of HPV. Several more recent studies have evaluated the effect of Zaprinast on pulmonary hemodynamics in the fetal-neonatal circulation. In the newborn lamb, Zaprinast increased endotheliumdependent vasodilation, consistent with augmentation of stimulated endothelium-derived nitric oxide (8). In that study, central venous administration of Zaprinast reduced Ppa during acute pulmonary vasoconstriction induced by either the thromboxane mimetic U46619 or hypoxia. While there was a tendency for relative pulmonary specificity, reduction in systemic pressure at submaximal Zaprinast concentrations, similar to those seen in the present study, were reported. The same group also found that L-arginine pretreatment attenuated neonatal pulmonary vasoconstriction (14), supporting the concept that augmentation of endogenous pulmonary NO production can attenuate pulmonary hypertension in the neonate. A recent preliminary report also showed that cGMP PDE inhibition dilates the ovine fetal pulmonary hypertensive circulation, 
though pulmonary selectivity could not be evaluated as drug was delivered directly into the left pulmonary artery (34).

Inhaled NO is currently the only clinically available specific pulmonary vasodilator. We therefore questioned whether the vasodilation in response to cGMP-PDE inhibition was quantitatively similar to that to inhaled NO. The maximum amount of pulmonary vasodilation and inhibition of HPV was similar between E4021, Zaprinast, and inhaled NO (up to $80 \mathrm{ppm}$ ). Of equal importance, the degree of pulmonary specificity was equivalent between E4021 and inhaled NO. While inhaled NO is not commonly used to treat chronic pulmonary hypertension, $\mathrm{Ca}^{2+}$ channel blockers are often used (35). We therefore tested the most commonly used agent in this class, diltiazem. In contrast to the NO/cGMP-dependent agents, diltiazem caused a profound degree of systemic hypotension, largely due to impaired cardiac output. This result is similar to that which we have previously reported with nifedipine (32), and suggests that in this model of chronic pulmonary hypertension, enhancement of NO/cGMP-mediated pulmonary vasodilation is superior to $\mathrm{Ca}^{2+}$ channel blockade.

The relevance of our observations to humans with pulmonary hypertension is speculative. In normal humans, inhibition of NOS results in increased pulmonary vascular resistance, confirming a role for NO (and presumably cGMP) in modulating pulmonary artery tone (26). In humans with pulmonary hypertension it is uncertain if NO production is increased, as it is in the rat. A recent report from human pulmonary hypertensive lungs removed at the time of transplantation found reduced NOS message and protein (36). The reason for the discrepancy between animal studies and this report in human tissue is uncertain, although the chronicity of the lesion (more prolonged and severe in the human transplant recipients) may play a role. In addition to alterations in the NO/cGMP system, circulating levels of atrial naturetic peptide, which can vasodilate the pulmonary circulation through stimulation of particulate guanylate cyclase, are increased in human primary and secondary pulmonary hypertension (37). Thus, even if NOS and NO are impaired in human lungs with established pulmonary hypertension, it is possible that augmentation of endogenous pulmonary cGMP levels by PDE5 inhibition would dilate the human pulmonary hypertensive circulation.

Our results establish the rationale for and demonstrate the potential utility of modulation of lung PDE activity as therapy for chronic pulmonary hypertension. We found that cGMP hydrolytic activity in pulmonary hypertensive rat lungs is predominantly cGMP-specific and that total lung cGMP hydrolytic activity was not significantly reduced, relative to normotensive controls. Therefore, elevations in lung cGMP in pulmonary hypertension appear to be mediated largely by increased guanylate cyclase activity, rather than impaired cGMP degradation. Further, we found that infusion of the cGMPspecific PDE inhibitor E4021 produced acute pulmonary vasodilation which was comparable to that seen with inhaled NO with respect to both maximum efficacy and pulmonary specificity in this model. Thus, it appears that despite increased endogenous NO and cGMP production in the pulmonary hypertensive circulation, significant additional vasodilation can be effected through further cGMP accumulation in response to PDE5 inhibition. While this study did not test the effect of chronic cGMP-specific PDE inhibition, our results suggest that such a strategy could provide a novel approach to therapy of pulmonary hypertension.

\section{Acknowledgments}

Special thanks to Lori E. Cohen for manuscript preparation, and Sandra Walchek for technical support.

The authors acknowledge the following grant support: American Heart Association Established Investigator Award 93002450 (D. M. Rodman), National Heart Lung and Blood Institute (NHLBI), HL48038 (D. M. Rodman), NHLBI 14985 (I. F. McMurtry), and National Institutes of Health institutional National Research Service Award grant HL-07670 (A. H. Cohen).

\section{References}

1. Fratacci, M., C. G. Frostell, T. Chen, J. C. Wain, D. R. Robinson, and W. M. Zapol. 1991. Inhaled nitric oxide a selective pulmonary vasodilator of heparin-protamine vasoconstriction in sheep. Anesthesiology. 75:990-999.

2. Rich, G. F., C. M. Roos, S. M. Anderson, D. C. Urich, M. O. Daugherty, and R. A. Johns. 1993. Inhaled nitric oxide: dose response and the effects of blood in the isolated rat lung. J. Appl. Physiol. 75:1278-1284.

3. Adnot, S., C. Kouyoumdjian, C. Defouilloy, P. Andrivet, S. Sediame, R. Herigault, and M. Fratacci. 1993. Hemodynamic and gas exchange responses to infusion of acetylcholine and inhalation of nitric oxide in patients with chronic obstructive lung disease and pulmonary hypertension. Am. Rev. Respir. Dis. 148:310-316.

4. Bigatello, L. M., W. E. Hurford, R. M. Kacmarek, J. D. Roberts, and W. M. Zapol. 1994. Prolonged inhalation of low concentrations of nitric oxide in patients with severe adult respiratory distress syndrome. Effects on pulmonary hemodynamics and oxygenation. Anesthesiology. 80:761-770.

5. Kinsella, J. P., and S. H. Abman. 1994. Efficacy of inhalation nitric oxide therapy in the clinical management of persistent pulmonary hypertension of the newborn. Chest. 105(Suppl.):92-94.

6. Kinsella, J. P., S. R. Neish, D. D. Ivy, E. Shaffer, and S. H. Abman. 1993. Clinical responses to prolonged treatment of persistent pulmonary hypertension of the newborn with low doses of inhaled nitric oxide. J. Pediatr. 123:103108.

7. Barst, R.J., L.J. Rubin, M.D. McGoon, E.J. Caldwell, W.A. Long, and P.S. Levy. 1994. Survival in primary pulmonary hypertension with long-term continuous intravenous prostacyclin. Ann. Intern. Med. 121:409-415.

8. Braner, D. A. V., J. R. Fineman, R. Chang, and S. J. Soiffer. 1993. M\&B22948, a cGMP phosphodiesterase inhibitor, is a pulmonary vasodilator in lambs. Am. J. Physiol. 264:H252-H258.

9. Tiktinsky, M. H., J. J. Cummings, and F. C. Morin, III. 1992. Acetylcholine increases pulmonary blood flow in intact fetuses via endothelium-dependent vasodilation. Am. J. Physiol. 262(Heart Circ. Physiol. 31):H406-H410.

10. Oka, M. K., K. Hasunuma, S. A. Webb, T. J. Stelzner, D. M. Rodman, and I. F. McMurtry. 1993. EDRF suppresses an unidentified vasoconstrictor mechanism in hypertensive rat lungs. Am. J. Physiol. 264(Lung Cell. Mol. Physiol. 8):L587-597.

11. Russ, R. D., and B. R. Walker. 1993. Maintained endothelium-dependent pulmonary vasodilation following chronic hypoxia in the rat. J. Appl. Physiol. 74:339-344.

12. Isaacson, T. C., V. Hampl, E. K. Weir, D. P. Nelson, and S. L. Archer. 1994. Increased endothelium derived NO in hypertensive pulmonary circulation of chronically hypoxic rats. J. Appl. Physiol. 76:933-940.

13. Shaul, P. W., A. M. North, T. S. Brannon, K. Ujiie, L. B. Wells, P. A. Nisen, C. J. Lowenstein, S. H. Snyder, and R. A. Star. 1995. Prolonged in vivo hypoxia enhances nitric oxide synthase type I and type III gene expression in adult rat lung. Am. J. Respir. Cell Mol. Biol. 13:167-174.

14. Fineman, J. R., R. Chang, and S. J. Soifer. 1992. EDRF inhibition augments pulmonary hypertension in intact newborn lambs. Am. J. Physiol. 262(Heart Circ. Physiol. 31):H1365-H1371.

15. Baudouin, S. V., P. Bath, J. F. Martin, R. DuBois, and T. W. Evans. 1993. L-arginine infusion has no effect on systemic haemodynamics in normal volunteers, or systemic and pulmonary haemodynamics in patients with elevated pulmonary vascular resistance. Br. J. Clin. Pharmacol. 36:45-49.

16. Surdacki, A., K. Zmudka, K. Bieron, E. Kostka-Trabka, J. S. Dubiel, and R. J. Gryglewski. 1994. Lack of beneficial effects of L-arginine infusion in primary pulmonary hypertension. Wien. Klin. Wochenschr. 106:421-526.

17. Popescu, L. M., C. Panoiu, M. Hinescue, and D. Nutu. 1985. The mechanism of cGMP-induced relaxation in vascular smooth muscle. Eur. J. Pharmacol. 107:393-394.

18. Schoeffler, P., C. Lugnier, F. Demesy-Waeldele, and J. C. Stodet. 1987. Role of cyclic AMP and cyclic GMP phosphodiesterases in the control of cyclic nucleotide levels and smooth muscle tone in rat isolated aorta. Biochem. Pharmacol. 36:3965-3972.

19. Souness, J. E., R. Brazdil, B. K. Diocee, and R. Jordan. 1989. Role of selective cyclic GMP phosphodiesterae inhibition in the myorelaxant acts of M\&B 22, 948, MY - 5445, vinpocetine and 1-methyl-3-isobutyl-8-(methylamino)xanthine. Br. J. Pharmacol. 98:725-734. 
20. Ahn, H. S., W. Crim, B. Pitts, and E. J. Sybertz. 1992. Calcium-calmodulin-stimulated and cyclic-GMP-specific phosphodiesterases: tissue distribution, drug sensitivity and regulation of cyclic GMP levels. In Advances in Second Messenger and Phosphoprotein Research. Vol. 25. S. J. Stradz and H. Hidaka, editors. Raven Press Ltd., New York. 271-288.

21. Zeigler, J. W., D. D. Ivy, J. J. Fox, J. P. Kinsella, W. R. Clarke, and S. H. Abman. 1995. Dipyridamole, a cGMP phosphodiesterase inhibitor, causes pulmonary vasodilation in the ovine fetus. Am. J. Physiol. In press.

22. Beavo, J. A., J. G. Hardman, and E. W. Sutherland. 1970. Hydrolysis of cyclic guanosine and adenosine $3^{\prime}, 5^{\prime}$-monophosphates by rat and bovine tissues. J. Biol. Chem. 245:5649-5655.

23. Bradford, M. M. 1976. A rapid and sensitive method for the quantitation of microgram quantities of protein utilizing the principle of protein-dye binding. Anal. Biochem. 72:248-254.

24. Hideyuki, A., T. Saeki, N. Mori, and I. Saito. 1994. Cardiohemodynamic characteristics of a novel, selective and potent phosphodiesterase V inhibitor, E4021, in the pig. JPN. J. Pharmacol. 64:329a. (Abstr.)

25. McMurtry, I. F., M. Oka, K. Hasunuma, J. Yamaguchi, K. Morris, and D. Rodman. Role of EDRF in control of normoxic and hypoxic pulmonary vascular tone. 1992. In High Altitude Medicine. G. Veda, J. T. Reeves, and M. Sekiguchi, editors. Shinshu University Press, Matsumoto-shi, Japan. 131-138.

26. Stamler, J. S., E. Loh, M. A. Roddy, K. E. Currie, and M. A. Creager. 1994. Nitric oxide regulates basal systemic and pulmonary vascular resistance in healthy humans. Circulation. 89:2035-2040.

27. McMahon, T. J., L. J. Ignarro, and P. J. Kadowitz. 1993. Influence of zaprinast on vascular tone and vasodilator responses in the cat pulmonary vascular bed. J. Appl. Physiol. 74:1704-1711.

28. Dundore, R. L., P. G. Habeeb, P. F. Pratt, L. T. Becker, D. M. Clas, and R. A. Buchholz. 1992. Differential hemodynamic responses to selective inhibitors of cyclic nucleotide phosphodiesterases in conscious rats. J. Cardiovasc. Pharmacol. 19:937-944.
29. Clarke, W. R., S. Vezono, A. Chambers, and P. Doepfner. The type III phosphodiesterase inhibitor milrinone and type V PDE inhibitor dipyridamole individually and synergistically reduce elevated pulmonary vascular resistance. Pulm. Pharmacol. 7:81-89.

30. Rosenkrantz, J. G., F. P. Lynch, and J. H. K. Vogel. 1972. Hypoxic pulmonary hypertension: its modification by dipyridamole. J. Surg. Res. 12:330333

31. Mlczoch, J., E. K. Weir, and R. F. Grover. 1977. Inhibition of hypoxic pulmonary vasoconstriction by dipyridamole is not platelet mediated. Can. J. Physiol. Pharm. 55:448-451.

32. Adachi, H., S. Yoshitake, and I. Saito. 1995. Cardiohemodynamic profile of E4021, a selective and potent phosphodiesterase type V inhibitor, in anesthestized pigs. 1994. JPN. J. Pharmacol. 64:329P.

33. Oka, M., K. G. Morris, and I. F. McMurtry. 1993. NIP-121 is more effective than nifedipine in acutely reversing chronic pulmonary hypertension. $J$. Appl. Physiol. 75:1075-1080.

34. Ziegler, J. W., D. D. Ivy, J. J. Fox, J. P. Kinsella, W. R. Clarke, and S. H. Abman. 1995. Hemodynamic effects of cGMP phosphodiesterase inhibitors during chronic pulmonary hypertension in the ovine fetus. Pediatr. Res. 37: 38a. (Abstr.)

35. Rich, S., E. Kaufmann, and P. S. Levy. 1992. The effect of high doses of calcium-channel blockers on survival in primary pulmonary hypertension. $N$. Engl. J. Med. 327:76-81.

36. Giaid, A., and D. Saleh. 1995. Reduced expression of endothelial nitric oxide synthase in the lungs of patients with pulmonary hypertension. $N$. Engl. J. Med. 333:214-221.

37. Morice, A. H., J. Pepke-Zaba, M. J. Brown, P. S. Thomas, and T. N. Higenbottom. 1990. Atrial natriuretic peptide in primary pulmonary hypertension. Eur. Respir. J. 3:910-913. 